Faculty of Economics and Social Sciences

Department of Economics

\title{
The Allocation of Resources under Uncertainty
}

Harris Dellas

Ana Fernandes

06-06

December 2006

\section{DISCUSSION PAPERS}




\title{
The Allocation of Resources under Uncertainty*
}

\author{
Harris Dellas ${ }^{\dagger} \quad$ Ana Fernandes ${ }^{\ddagger}$
}

\begin{abstract}
We study the effects of uncertainty on the allocation of resources in the standard, static, general equilibrium, two-sector, two-factor model. The elasticity of substitution in production vs that in consumption plays a key role in determining whether uncertainty attracts or repels resources. Risk aversion matters, but to a smaller extent, while factor endowments and factor intensities play a more limited role.
\end{abstract}

JEL class: E2, D5, D8

Keywords: Uncertainty, general equilibrium, two-sector model.

${ }^{*}$ We thank Michele Boldrin, Ron Jones, Bob King, Bob Lucas, Sergio Rebelo and Anne Sibert for useful conversations.

${ }^{\dagger}$ Department of Economics, University of Bern, CEPR, IMOP. Address: VWI, Schanzeneckstrasse 1, CH 3012 Bern, Switzerland. Tel: (41) 31-6313989, Fax: (41) 31-631-3992, email: harris.dellas@vwi.unibe.ch, Homepage: http://www.vwi.unibe.ch/amakro/

${ }^{\ddagger}$ :Department of Economics, University of Bern. Address: VWI, Schanzeneckstrasse 1, CH 3012 Bern, Switzerland. Tel: (41) 31-6315599, Fax: (41) 31-631-3992, email: ana.fernandes@vwi.unibe.ch, Homepage: http://anafernandes.name 


\section{Introduction}

Research on the effects of uncertainty on the allocation of resources goes back a long way. Writing in 1971, Rothemberg and Smith, classified the existing research into two groups. One included the general equilibrium models under complete Arrow-Debreu markets and concentrated almost exclusively on questions of welfare. As Rothemberg and Smith remarked "... using a very general framework, they (these authors) have examined the conditions under which a competitive equilibrium with futures markets will in some sense be optimal. They have not examined the comparative statics question concerning how changes in the amount of uncertainty affect the equilibrium prices and quantities traded." (p. 441). The second group consisted of "...partial equilibrium models that concentrate on the individual firm or individual consumer, who is assumed to optimize facing given prices... "The Markovitz-type portfolio analysis, as well as the theory of the firm under uncertainty (for instance, Oi, 1961), are examples of this approach.

Rothemberg and Smith set out to fill this gap by studying the effects of uncertainty on resource allocation in the standard, static, general equilibrium, competitive, twofactor, two-good model. However, in spite of the pioneering nature and importance of their work, they fell short of their stated objectives as they too ended up assuming given prices, instead of allowing them to be endogenously determined in general equilibrium.

Remarkably, the state of affairs concerning uncertainty and resource allocation has not advanced much since Rothemberg and Smith's paper. In other words, there exists no work establishing how uncertainty affects the allocation of resources in the basic $2 X 2$ model. Such a claim may appear incredulous in the face of two influential, distinct literatures that have dealt with uncertainty in general equilibrium. Namely, the theory of international trade under uncertainty (for instance, Anderson, 1981, Eaton, 1979, Helpman and Razin, 1978), and the real business cycle theory (King and Rebelo, 2000). Nonetheless, neither of these literatures has really addressed this issue.

The international trade literature has been exclusively concerned with the questions of a) whether the presence of uncertainty undermines comparative advantage as the 
basis for trade and, b) whether and under what conditions the standard propositions of trade (Heckscher-Ohlin theorem $(\mathrm{H}-\mathrm{O})$, international factor price equalization, and so on) still hold under uncertainty. These questions are undoubtedly important but are only partially related to the issue of how uncertainty affects the allocation of resources. For instance, a finding that the $\mathrm{H}-\mathrm{O}$ theorem still holds under uncertainty does not suffice to determine the direction in which the production pattern is shifted as a result of uncertainty. ${ }^{1}$

The real business cycle literature, on the other hand, has typically employed models where certainty equivalence holds (typically by using a linear approximation around the deterministic steady state). Thus, by construction, it has shunned away from dealing with the effects of uncertainty on average (long term) allocations.

The objective of this paper is to study the effects of uncertainty on the allocation of resources in the standard, $2 X 2$ model with complete asset markets. Our approach differs from Rothemberg and Smith in two important respects. First, we use a proper general equilibrium model in the sense that we allow prices to be endogenously determined alongside quantities. And second, we include a demand side to the model so that consumer preferences play a critical role in the allocation of resources. Rothemberg and Smith, on the other hand, took prices for granted, focused exclusively on the producers, and used GDP as their welfare criterion. ${ }^{2}$

We derive the effects of uncertainty on production patterns as a function of preference and production parameters, the distribution of uncertainty across sectors, and the overall supplies of the factors of production. Using an approximation to the optimality conditions, we manage to fully characterize the behavior of the economy and to obtain results in terms of the key parameters of the model.

The rest of the paper is organized as follows. Section 1 sets up the model. Section 2 reports the comparative statics exercises. Section 3 concludes.

\footnotetext{
${ }^{1}$ An additional problem with the trade literature is that it has only managed to address special cases of uncertainty.

${ }^{2}$ Another difference concerns the timing of the decisions in relation to the resolution of uncertainty. We treat the Rothemberg and Smith assumption of labor decisions being made after the resolution of uncertainty as a special case.
} 


\section{The model}

The model is the standard, static, perfectly competitive, closed economy, two-good, two-factor model, with a representative agent and complete asset markets.

Utility is derived from consumption of two goods, $X$ and $Y$, in amounts $c_{x}$ and $c_{y}$ respectively:

$$
U=U\left(C\left(c_{x}, c_{y}\right)\right)
$$

where $C$ is a consumption aggregate. The utility function has standard properties.

Production of $X$ and $Y$ satisfies:

$$
X=F\left(A_{x}, L_{x}, K_{x}\right), \quad Y=G\left(A_{y}, L_{y}, K_{y}\right)
$$

where $F$ and $G$ have standard properties. $L_{i}, K_{i}, i=x, y$ are the amounts of labor and capital employed in the $X$ and $Y$ sectors, respectively. $A_{x}$ and $A_{y}$ are random variables representing variation in the state of technology in these two sectors.

The factor allocations satisfy:

$$
\begin{aligned}
L_{x}+L_{y} & =L \\
K_{x}+K_{y} & =K,
\end{aligned}
$$

where $K$ and $L$ are fixed.

Let $s_{l x} \equiv L_{x} / L, s_{k x} \equiv K_{x} / K$. A social planner selects $\left\{s_{l x}, s_{k x}\right\}$ in order to maximize (1) subject to (2)-(4). In order to examine the effects of uncertainty, we first need to know the allocations under certainty. For now, let $A_{x}=A_{y}=A$. First order conditions are:

$$
\begin{gathered}
\frac{d C}{d c_{x}} \frac{d c_{x}}{d s_{l x}}-\frac{d C}{d c_{y}} \frac{d c_{y}}{d s_{l y}}=0 \\
\frac{d C}{d c_{x}} \frac{d c_{x}}{d s_{k x}}-\frac{d C}{d c_{y}} \frac{d c_{y}}{d s_{k y}}=0
\end{gathered}
$$

where $s_{l y}=1-s_{l x}$ and $s_{k y}=1-s_{k x}$. Equations (5)-(6), together with the production functions and the equilibrium relations $c_{x}=X$ and $c_{y}=Y$, determine the optimal levels of $X$ and $Y$ under certainty, $\bar{X}$ and $\bar{Y}$. 


\section{Uncertainty}

We now introduce uncertainty. We assume that the production decisions, namely the allocation of labor and capital across sectors by the social planner, are made before the resolution of uncertainty. Once the sectorial allocations of labor and capital have been determined, uncertainty is resolved and production ensues. After that, consumption takes place.

The optimality conditions now take the form:

$$
\begin{gathered}
E \frac{d U}{d C}\left(\frac{d C}{d c_{x}} \frac{d c_{x}}{d s_{l x}}-\frac{d C}{d c_{y}} \frac{d c_{y}}{d s_{l y}}\right)=0 \\
E \frac{d U}{d C}\left(\frac{d C}{d c_{x}} \frac{d c_{x}}{d s_{k x}}-\frac{d C}{d c_{y}} \frac{d c_{y}}{d s_{k y}}\right)=0,
\end{gathered}
$$

where $E$ denotes the expectations operator with respect to the distribution of output shocks, $A_{x}$ and $A_{y}$.

The term inside the parenthesis in (7) represents the "excess return" in sector $X$, measured in terms of total consumption. If this excess return co-varies positively with total consumption, then allocating resources to the $X$ sector is a risky enterprise. In such a case, the $X$ sector will repel resources relative to the case of certainty. The opposite pattern obtains if it co-varies negatively. If the covariance is zero, then uncertainty has no effect on the allocation of resources. A sufficient condition for the covariance to be zero is for the term inside the parenthesis not to involve aggregate consumption. This condition is satisfied when the consumption aggregator $C$ is logarithmic and the production function is Cobb-Douglas (C-D).

In general, more structure needs to be imposed in order to determine the effects of uncertainty. We consider a Constant Elasticity of Substitution (CES) specification for utility and production.

The utility function is now:

$$
U=\frac{1}{1-\gamma} C^{1-\gamma}=\frac{1}{1-\gamma}\left[\left(w_{c} c_{x}^{\rho}+\left(1-w_{c}\right) c_{y}^{\rho}\right)^{\frac{1}{\rho}}\right]^{1-\gamma}
$$


with

$$
\rho \in(-\infty, 1], \gamma \geq 0,0<w_{c}<1
$$

whereas the production functions are specialized to:

$$
\begin{aligned}
& c_{x}=X=A_{x}\left[w_{x}\left(s_{x} L\right)^{\theta}+\left(1-w_{x}\right)\left(s_{k x} K\right)^{\theta}\right]^{\frac{1}{\theta}} \\
& c_{y}=Y=A_{y}\left[w_{y}\left(s_{l y} L\right)^{\theta}+\left(1-w_{y}\right)\left(s_{k y} K\right)^{\theta}\right]^{\frac{1}{\theta}},
\end{aligned}
$$

with

$$
\theta \in(-\infty, 1], 0<w_{i}<1, i=x, y
$$

Equations (7)-(8) now take the form:

$$
\begin{gathered}
E U_{l x}=E\left\{C^{1-\gamma-\rho}\left(c_{x}^{\rho-\theta} w_{c} w_{x} s_{l x}^{\theta-1}-c_{y}^{\rho-\theta}\left(1-w_{c}\right) w_{y} s_{l y}^{\theta-1}\right)\right\}=E\left(Z * W_{l}\right)=0 \\
E U_{k x}=E\left\{C^{1-\gamma-\rho}\left(c_{x}^{\rho-\theta} w_{c}\left(1-w_{x}\right) s_{k x}^{\theta-1}-c_{y}^{\rho-\theta}\left(1-w_{c}\right)\left(1-w_{y}\right) s_{k y}^{\theta-1}\right)\right\}=E\left(Z * W_{k}\right)=0
\end{gathered}
$$

Note that the fact that $s_{l x}$ and $s_{k x}$ are selected (known) before the resolution of uncertainty implies that these two first-order conditions can be combined to give:

$$
\frac{w_{x}}{1-w_{x}}\left(\frac{s_{l x}}{s_{k x}}\right)^{\theta-1}=\frac{w_{y}}{1-w_{y}}\left(\frac{s_{l y}}{s_{k y}}\right)^{\theta-1} .
$$

Therefore, $s_{k x}$ is a deterministic function of $s_{l x}$ and knowledge of the latter suffices to characterize the behavior of the model under uncertainty. This implies also that, in the case of the CES specification, an alternative timing with capital decisions being made before the resolution of uncertainty and labor decisions after the resolution would not make any difference for our results.

If the elasticities of substitution in production and consumption are equal, $\rho=\theta$, then $W_{l}$ is independent of $X$ and $Y$ and uncertainty does not matter for the optimal allocation of resources. The special case of $\rho=\theta=0$ corresponds to a logarithmic consumption aggregator $C$ and Cobb-Douglas production functions.

The situation is more complicated when $\rho \neq \theta$. Let us rewrite $E U_{l x}$ as 


$$
E U_{l x}=E\left(Z * W_{l}\right)=E Z * E W_{l}+\operatorname{cov}\left(Z, W_{l}\right)=0
$$

where $W_{l}$ is the term inside the parenthesis in (11). Since $E Z>0, \operatorname{sign}\left(E W_{l}\right)=$ $-\operatorname{sign}\left(\operatorname{cov}\left(Z, W_{l}\right)\right)$.

In order to determine how $s_{l x}$ varies with the introduction of -or an increase inuncertainty, we need to know how $E W_{l}$ and $\operatorname{cov}\left(Z, W_{l}\right)$ vary with uncertainty as well as with $s_{l x}$. Knowledge of how $E W_{l}$ and $\operatorname{cov}\left(Z, W_{l}\right)$ vary with uncertainty -for a given value of $s_{l x}$-allows us to determine how the first-order condition is perturbed away from satisfying $E U_{l x}=0$. Knowledge of how they vary with $s_{l x}$ allows us to determine how to change $s_{l x}$ in order to restore (14).

We start by discussing the properties of $W_{l}$. It is instructive to assume (as in Rothemberg and Smith) that only one sector is subject to uncertainty. We set $A_{y}=1$. In fact, the more general case turns out to be quite intractable from an intuitive point of view, except for the case when uncertainty is of the of aggregate form only (the sectorial shocks are perfectly correlated).

Proposition 1. $W_{l}$ is a decreasing function of $s_{l x}$.

Proof. $W_{l}=X^{\rho-1} F^{\prime}-Y^{\rho-1} G^{\prime}$, where $F^{\prime}=d X / d s_{l x}$ and $G^{\prime}=d Y / d s_{l y}$. Consequently, $d W_{l} / d s_{l x}=(\rho-1) X^{\rho-2}\left(F^{\prime}\right)^{2}+X^{\rho-1} F^{\prime \prime}+(\rho-1) Y^{\rho-2}\left(G^{\prime}\right)^{2}+Y^{\rho-1} G^{\prime \prime}<0$, where $F^{\prime \prime}=d F^{\prime} / d s_{l x}$ and $G^{\prime \prime}=d G^{\prime} / d s_{l y}$.

Proposition 2. $W_{l}$ is a concave (convex) function of $A_{x}$ if $(\rho-\theta)(\rho-\theta-1)<(>) 0$. Proof.

$$
\operatorname{sign}\left(\frac{d^{2} W}{d A_{x}^{2}}\right)=\operatorname{sign}((\rho-\theta)(\rho-\theta-1)) .
$$

The following result follows directly from Jensen's inequality.

Proposition 3. If $W_{l}$ is a concave (convex) function of $A_{x}$, then $E W_{l}<0(>0)$ in the neighborhood of the deterministic equilibrium, $\bar{X}$ and $\bar{Y}$.

These three propositions, together with (14), can now be used to study how uncertainty in the $X$ sector affects the optimal choice of $s_{l x}$. Let $\bar{s}_{l x}$ denote the optimal labor 
share allocated to sector $X$ under certainty and $s_{l x}^{*}$ stand for the corresponding optimal value under uncertainty.

\section{Proposition 4.}

(a) Suppose $\operatorname{cov}\left(Z, W_{l}\right) \leq 0$. If $W_{l}$ is a concave function of $A_{x}$, then $s_{l x}^{*}<\bar{s}_{l x}$.

(b) Suppose $\operatorname{cov}\left(Z, W_{l}\right) \geq 0$. If $W_{l}$ is a convex function of $A_{x}$, then $s_{l x}^{*}>\bar{s}_{l x}$.

Proof. (a) From the concavity of $W_{l}, E W_{l}<0$ when evaluated at $s_{l x}=\bar{s}_{l x}$. Since $E Z>0$, it follows that $E U_{l}<0$. For optimality to be restored, $s_{l x}$ must decrease relative to $\bar{s}_{l x}$. Part (b) follows from identical reasoning ${ }^{3}$.

Proposition 2 relates the curvature of $W_{l}$ to the parameters of the model. The sign of the covariance can be similarly related to the parameters of the model. Taking a first order approximation to $Z$ and $W_{l}$ around the deterministic equilibrium, and then using the resulting expressions to compute the covariance gives :

$$
\operatorname{sign}(\operatorname{cov})=\operatorname{sign}((\rho-\theta)(1-\rho-\gamma))
$$

Combining propositions (3)-(4) with equation (16) leads to the results summarized in Table 1.

Table 1: The main patterns

$$
\begin{aligned}
& s_{l x} \downarrow \quad \text { if } \quad\{(\rho-\theta)(1-\rho-\gamma) \leq 0 \&(\rho-\theta)(\rho-\theta-1)<0\} \\
& s_{l x} \uparrow \quad \text { if } \quad\{(\rho-\theta)(1-\rho-\gamma) \geq 0 \&(\rho-\theta)(\rho-\theta-1)>0\}
\end{aligned}
$$

Note that the only parameters that matter for determining the effects of uncertainty are $\rho, \theta$, and $\gamma$; in particular, the capital-to-labor ratio of the economy plays no role. ${ }^{4}$

\footnotetext{
${ }^{3}$ Note that the value -and its variation- of $s_{l x}$ matters for the size but not for the sign of the covariance.

${ }^{4}$ It should also be mentioned that the conditions for an activity (asset) to attract or repel resources are different from those obtained under partial equilibrium. In the latter case the rate of return from pursuing a particular activity (investing in a particular asset) is independent of the scale of investment. Therefore, the parameter $\theta$ no longer enters the allocation decision.
} 
Intuition While it is difficult to offer an intuition in the general case because of the large number of parameters and parameter configurations, there are special cases of interest that are more amenable to economic interpretation. Consider for instance the case of $\theta=0$ (C-D production functions). If the goods are good-substitutes $(\rho>0)$, and the agents are sufficiently risk averse $(\gamma>1)$, then exposing resources to uncertainty is not a good idea because it destabilizes the aggregate consumption bundle without bringing any significant benefits regarding the smoothness of the individual components of consumption. Recall that the greater the degree of substitutability between $X$ and $Y$, the greater the dependence of the marginal utility of $X$ and $Y$ on their sum, $X+Y$, rather than on $X$ and $Y$ individually. In this case, equation (15) implies that $W$ is concave $(\rho(\rho-1)<0)$, and equation (16) that the covariance is negative $(\rho(1-\rho-\gamma)<0)$. If, on the other hand, the two goods are poor substitutes $(\rho<0)$, then individual good consumption smoothing becomes important relative to the smoothing of the aggregate bundle. Low realizations of the stochastic output are costly and the agents attempt to get insurance against this by making a bigger investment in the sector facing uncertainty.

The situation is more complicated when $(\rho-\theta)(1-\rho-\gamma)$ and $(\rho-\theta)(\rho-\theta-1)$ have opposite signs. ${ }^{5}$ That is, when either $\operatorname{cov}\left(Z, W_{l}\right)<0$ and $W_{l}$ is a convex function of $A_{x}$, or when $\operatorname{cov}\left(Z, W_{l}\right)>0$ and $W_{l}$ is a concave function of $A_{x}$. The optimal choice of $s_{l x}$ cannot be determined without additional steps. One possible approach is to also approximate $Z$ and $W_{l}$ in the neighborhood of the deterministic equilibrium and compute $E U_{l}=E Z * E W_{l}+\operatorname{cov}\left(Z, W_{l}\right)$ based on this approximation. Due to the fact that $E W_{l}=0$ when evaluated at the deterministic solution, we need to use -at least- a second order approximation to $W_{l}$. Therefore, taking a linear approximation to $E Z$ and a quadratic one to $E W$ leads to

$$
\operatorname{sign}\left\{E U_{l x}\right\}=\operatorname{sign}\{(\rho-\theta)[(1-\rho-\gamma) \Delta-0.5(1-\rho+\theta)]\},
$$

\footnotetext{
${ }^{5}$ Note that this statement is equivalent to $(1-\rho-\gamma)(1-\rho+\theta)>0$.
} 
where

$$
\Delta \equiv \frac{1}{1+(\bar{Y} / \bar{X})^{\rho}}<1
$$

In this case, factor endowments and factor intensities matter for the effects of uncertainty through their influence on $\Delta$. In particular, if $Y$ is intensive in the "abundant" factor, ${ }^{6}$ then $\Delta<0.5(>0.5)$ when $\rho>0(<0)$. Conversely, if $X$ is intensive in the "abundant" factor then $\Delta>0.5(<)$ when $\rho<0(>0)$.

Proposition 6. $U_{l x}$ is a decreasing function of $s_{l x}$.

Proof: This follows immediately from satisfaction of the second order condition of the optimization problem.

Proposition 6 implies that if -the approximate $-E U_{l x}$ is negative (positive), $s_{l x}$ must be decreased (increased) in order to satisfy the first-order condition for optimality.

As seen earlier, if both $(\rho-\theta)((1-\gamma-\rho)$ and $(\rho-\theta)(\rho-\theta-1))$ are negative (positive), then uncertainty in $X$ decreases (increases) $s_{l x}$. This is the pattern already established earlier in Table 1. When these two expressions are of opposite sign, however, the effects of uncertainty depend on the absolute value of these two expressions as well as on the size of $\Delta$ relative to 0.5 . While the optimal allocations can be characterized for any parameter configuration of the model, it is difficult to provide generalizations. Nonetheless, some interesting patterns can be reported in the special case when $\bar{Y} / \bar{X}=1$ (the sectors have the same size under certainty). In this case, better substitutability in production relative to consumption $(\theta>\rho)$ tends to favor the risky sector. Similarly, high risk aversion favors (discourages) investment in the risky sector when substitutability in production is higher (lower) than that in consumption $(\theta>\rho)$.

\subsection{Extensions}

Uncertainty in both sectors The case of uncertainty in both sectors can be treated in a manner analogous to that of the previous section. Unfortunately, the approximated

\footnotetext{
${ }^{6}$ We associate abundance with a factor ratio greater than unity. This is not the definition used in trade theory but here there is only one country. The definition of factor intensity is the standard one. That is, industry $\mathrm{X}$ is capital intensive if $K_{x} / L_{x}>K_{y} / L_{y}$ at any wage-capital rental rate ratio.
} 
version of the optimality condition is not particularly revealing for gaining any insights into the effects of uncertainty on the pattern of production. ${ }^{7}$

Nonetheless, there is a special case of both theoretical and practical importance, that can easily be studied. Namely, the case of aggregate uncertainty, where shocks to both sectors are perfectly correlated. It can be easily verified that, in this case, $E W_{l}=0$, and thus the allocations under uncertainty are identical to those under certainty.

Labor decisions after the resolution of uncertainty This assumption does not matter as long as the relationship between $s_{l x}$ and $s_{k x}$ is independent of uncertainty. This is the case under a Cobb-Douglas or a CES production function.

\section{Conclusions}

We have analyzed the effects of uncertainty on the optimal allocation of resources in a general equilibrium, two-good, two-factor model with complete asset markets. This task has been long overdue as, surprisingly, there exists no treatment of this issue in the literature. The difference in the degree of flexibility (elasticity of substitution) in consumption relative to that in production turns out to play the key role. Risk aversion also matters, but is not necessary for uncertainty to alter the allocation of resources as uncertainty influences the allocation of resources even under risk neutrality. Factor endowments and factor intensities may also matter, but there exist situations in which they play (qualitatively ${ }^{8}$ ) no role.

\section{References}

Anderson, J., 1981, The Heckscher-Ohlin and Travis-Vanek theorems under uncertainty, Journal of International Economics, (11), 237-249.

Eaton, J., 1979, The allocation of resources in an open economy with uncertain terms

\footnotetext{
${ }^{7}$ It should be kept in mind that, for any parametrization of the model, it is always feasible to also solve the model numerically and derive the sectorial factor shares and levels of output.

${ }^{8}$ That is in determining whether uncertainty attracts or repels. They always matter for the size of the effects.
} 
of trade, International Economic Review, 20 (2), 391-403.

Helpman, E. and A. Razin, 1978, A theory of international trade under uncertainty, New York: Academic Press.

King, R. and S. Rebelo, 1999, Resuscitating real business cycles, Handbook of macroeconomics, Volume 1B, 1999, pp. 927-1007. New York: Elsevier Science, North-Holland. Levhari, D., 1972, Optimal savings and portfolio choice under uncertainty, in Szego and Shell (eds), Mathematical methods in investment and finance, North Holland.

Oi, W. 1961, The desirability of price instability under perfect competition, Econometrica, $29,1,58-64$.

Rothemberg, T., and K. Smith, 1971, The effect of uncertainty on the resource allocation in a general equilibrium model, Quarterly Journal of Economics, 85(3), 440-454. 